Original Article

\title{
Patterns of scrotal pain in a hospital setting
}

\author{
Chapagain S, Subedi PP, Thakur DK, Poudel S, Luitel BR, Chalise PR, Sharma UK, Gyawali PR, Shrestha GK
}

Department of Surgery, Tribhuvan University Teaching Hospital, Kathmandu, Nepal

Correspondence: Suman Chapagain

Email: drsumanchapagain@hotmail.com

\begin{abstract}
Introduction: Scrotal pain is a common urological condition. Common causes of scrotal pain are testicular torsion, epididymitis, epididymo-orchitis and occasionally due to post vasectomy pain, varicocele, testicular trauma etc. The objective of our study is to review the different disease patterns in patients presenting with scrotal pain in a tertiary care hospital.

Methods: Patients presenting with scrotal pain to the out-patient clinic and the emergency department were included in the study. All patients were subjected to urinalysis and ultrasound of scrotum with color Doppler study when needed. Descriptive variables were studied and analyzed.

Results: Eighty three patients were enrolled in the study during the period of one year. Age ranged from 13 to 72 years; most of the patients were less than 40 years and more than 20 years. Among this age group non-specific orchalgia was very common. Isolated epididymitis was seen in only 4 (4.81\%) patients. Epididymo-orchitis was found in $16(19.27 \%)$ patients. Torsion of testis was found in 6 patients $(7.22 \%)$. The major bulk of the patients presenting with scrotal pain were nonspecific which was found in $39(46.98 \%)$ and exact cause could not be found in them. Total of $15(18 \%)$ patients had acute presentation.
\end{abstract}

Conclusion: Management of scrotal pain can be simple or at times organ threatening, proper diagnosis and treatment by clinical judgment and necessary investigation is very important.

\section{Introduction}

Scrotal pain is one of the most common causes for patients visiting the urological outpatient clinics. Patients present with myriad of causes which leads to scrotal pain. Causes of scrotal pain can be inflammatory, traumatic or neoplastic; however clinically it can be acute or chronic. ${ }^{1}$ Important causes of scrotal pain are testicular torsion, epididymitis and epididymo-orchitis. Other causes are post vasectomy pain, varicocele, testicular trauma, tumor, non-urological causes like incarcerated hernia, peritonitis, ruptured aortic aneurysm, and referred scrotal pain and non-surgical causes like Henoch- Schonlein purpura. ${ }^{1}$

Because of these varied presentations and etiologies evaluation of such a patient can be a diagnostic dilemma, as physical exam findings may be nonspecific. However, most of these causes of scrotal pain can be diagnosed using ultrasound with or without color Doppler. ${ }^{2}$ Chronic orchalgia is another important condition which can be defined as intermittent or constant testicular pain for a period of three or more months that interferes with daily activities. ${ }^{3}$

Patients presenting with testicular pain are often a challenge to the urologists. Diagnosis should be made promptly and condition needs to be managed as soon as possible to prevent the stress, anxiety, morbidity and also at times to exclude fertility-threatening conditions like torsion of testis which needs immediate surgical management. ${ }^{4}$

Evaluation of different causes includes full history, physical examination and investigations. Among the investigations ultrasonography of scrotum with or without color Doppler study is the most sensitive tool. Acute form of scrotal pain is often an emergency even in a non-traumatic setting, a big concern being testicular torsion. ${ }^{4}$ 
Spectrum of the disease involving the testes are one of the least studied topic in most of the places. This study was designed to see the spectrum of orchalgia in a tertiary care hospital setting in Nepal.

\section{Methods}

This is a prospective observational study done at Tribhuvan University Teaching Hospital for a period of 6 months. It included all patients presenting to the hospital with history of scrotal pain.

Patients with other diagnoses like varicocele, primary hydrocele, inguinal hernia and superficial scrotal infection, post-vasectomy pain, post hernia surgery pain and trauma were excluded. All the patients who were included in the study were subjected to clinical examination and investigations. The work up included history, investigations urinalysis (urine routine and microscopic examination and culture) and ultrasonography (USG) of the scrotum with or without color Doppler study. Diagnoses were based on the clinical judgment and the impression of ultrasound done by the radiologist.

Patients after being diagnosed with the causes of scrotal pain were managed with the standard protocol which included antibiotics (Fluoroquinolone-Ciprofloxacin for 7 days), analgesics (combination of analgesic and antiinflammatory drug, Ibuprofen and Paracetamol for 3 days), and $\mathrm{H}_{2}$ receptor blocker (Ranitidine $150 \mathrm{mg} \mathrm{BD}$ for 7 days), along with scrotal support, or immediate surgical exploration on suspicion of torsion of testis. The descriptive variables were compared with the findings noted in the ultrasonography and results were analyzed. Data were entered in the excel spreadsheet and analyzed.

\section{Results}

Eighty three patients were included in the study out of them $12(14.45 \%)$ patients had history of sexual promiscuity. One patient $(1.2 \%)$ had persistent pain in-spite of treatment and on further evaluation was found to have tuberculosis.

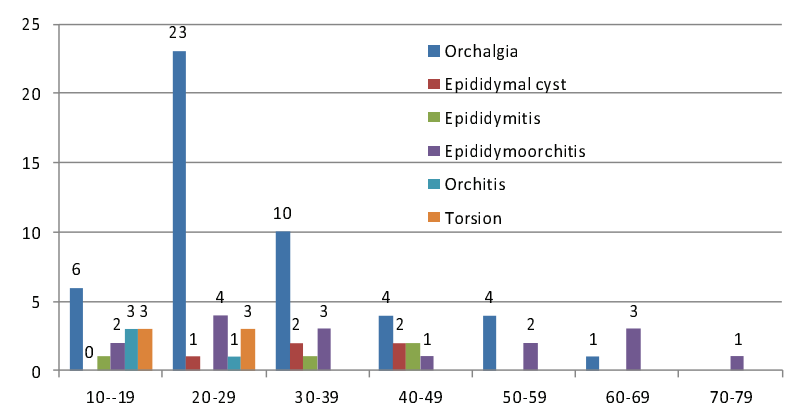

Figure 1: Distribution of patients with scrotal pain
Age of the patients who presented to the urology clinic or emergency with complaints of orchalgia ranged from 13 to 72 years. However most were between 20 to 40 years. Amongst this age group non-specific orchalgia was very common followed by epididymo-orchitis. (Figure 1)

Though patients presenting to the hospital were from all over the country, as expected, most were from Kathmandu (44.6\%) followed by Nuwakot. Most of the patients were from central and western part of the country. (Figure 2)

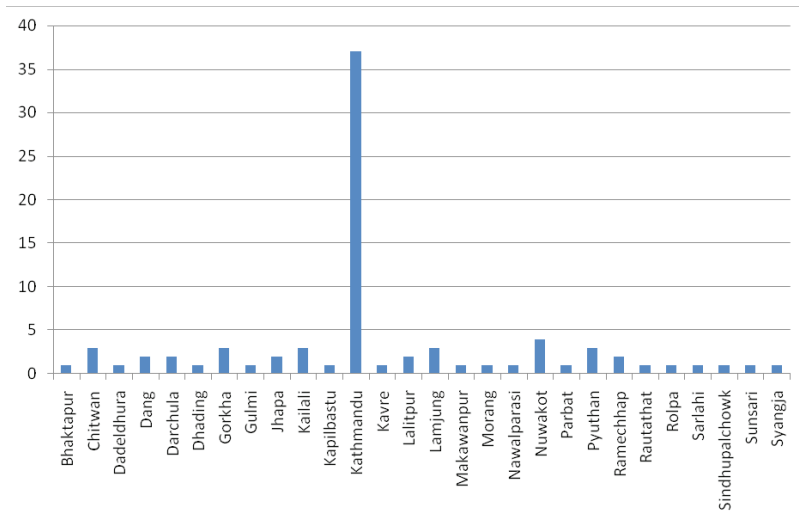

Figure 2: Geographical distribution of the patients

Table 1: Age group and side involved

\begin{tabular}{cccc|} 
Age & Right & Left & Bilateral \\
$10-19$ & 9 & 6 & 0 \\
$20-29$ & 11 & 15 & 6 \\
$30-39$ & 2 & 10 & 4 \\
$40-49$ & 5 & 3 & 1 \\
$50-59$ & 2 & 1 & 3 \\
$60-69$ & 1 & 0 & 3 \\
$70-79$ & 0 & 1 & 0 \\
Total & 30 & 36 & 17 \\
\hline
\end{tabular}

Most of the patients had left side involvement (43.37\%) and interestingly 17 patients $(20.48 \%)$ had bilateral testicular involvement. (Table 1)

Among the patients whose urine culture was positive the organism grown was E. coli; the growth was however seen only in 10 cases $(12 \%)$. The remaining $88 \%$ of the patients did not show growth in the culture. Of the patients with positive urine culture 5 patients showed bilateral involvement, 3 with orchalgia and 2 with epididymo- 
orchitis. Of the remaining 4 culture positive cases were orchalgia and one epididymo-orchitis.

Based on the USG scrotum findings, isolated epididymitis was found in only four patients (4.81\%) however, combined epididymo-orchitis was present in 16 (19.27\%) patients; two patients out of those were having urinary tract infection as well. Left side was involved in 6 patients of which one was tubercular and right sided involvement was seen in six patients.

The major bulk of the patients presenting with orchalgia were nonspecific, 39 patients $(46.98 \%)$ and exact cause could not be identified in them. Among our patients 42 $(50.6 \%)$ were married at the time of presentation. Swelling was present in $20(24 \%)$ patients and in 39\% the cause of orchalgia was idiopathic. Acute presentation was seen in $61(73.5 \%)$ and chronic presentation was seen in 22 $(26.5 \%)$.

\section{Discussion}

Of the total 83 patients most of the patients presenting with orchalgia were in between 20 to 40 years, however it included patients from 13 to 72 years.

Patients coming to the hospital with complaints were from all over the country including all the regions; maximum number of patients were from Kathmandu (44.6\%) as it is the capital city.

Among many diagnoses, torsion of testis was found in six $(7.22 \%)$ patients. In patients with torsion, 3 patients presented in the same day, within hours of onset of pain and testis was salvaged with fixation of contralateral testis in them. The other three had late presentation after 2 days and testis had to be removed. On suspicion of testicular torsion all the patients were taken for exploration, color Doppler study was done in all cases on the way to the operation theater. We found testicular torsion in younger age group range of 13-21 years, similar to the study reported from University of Missouri-Columbia School of Medicine. ${ }^{5}$ Testicular torsion is a medical emergency, requiring prompt treatment or risking the loss of the testicle. The incidence is 1 in 4,000 males under the age of 25 years. ${ }^{5}$

A positive urinalysis and urine cultures, along with leukocytosis, favor a diagnosis of epididymitis but do not exclude torsion. Color Doppler ultrasound or nuclear scintigraphy to assess blood flow to the scrotum and its contents helps to differentiate between the two entities. Doppler ultrasound would show increased blood flow, in an inflammatory condition. ${ }^{6}$ However, in torsion, vascularity may be compromised or even it may be absent. These findings were consistent with the findings obtained in patients in our study.

The major bulk of the patients presenting with scrotal pain were nonspecific: 39 patients $(46.98 \%)$ and exact cause could not be identified. Fifteen out of eighty three patients were found to have acute presentation comprising $61(73.5 \%)$ of the patients. Chronic orchalgia is a common and frustrating problem that most urologists will encounter frequently during their practice. ${ }^{3}$

Chronic orchalgia was seen in $22(26.5 \%)$ patients. It is a condition which has frequently idiopathic etiology, psychosocial impact, and treatment dilemma which often lead to patient distress, physician frustration, and incomplete care. It is defined as intermittent or constant testicular pain for a period of 3 or more months that interferes with daily activities. ${ }^{3}$ Pain may involve any of the scrotal contents including the testicle, epididymis, paratesticular structures, and spermatic cord. ${ }^{8}$ The etiology of orchalgia remains largely unknown in up to $50 \%$ of patients. ${ }^{1}$ This is similar to our results where $39 \%$ of patients were having idiopathic etiology.

Our study showed urine culture positive in only $12 \%$ of cases, rest of the cases did not show any organism which was similar to other study where it was shown that patients may present with scrotal pain and urinalysis may be normal in up to $50 \%$ of patients with epididymitis or orchitis. ${ }^{8}$

All the patients who were enrolled in the study were subjected to ultrasonographic evaluation of the scrotum. Among the patients 33 (39.75\%) had normal findings not corresponding to any disease pattern and 50 (60.24\%) had abnormal findings corresponding to the diagnosis.

It is important to point out that epididymitis may be isolated to the body or the tail and can be missed if these structures are not identified and evaluated with USG. On USG, epididymitis or orchitis is expected to show significantly increased blood flow (hyperemia) compared with the normal testis and epididymis. There can be significant hydrocele secondary to the inflammation. ${ }^{7,10,11,12}$ Among patients with orchalgia, 12 (14.45\%) patients had hydrocele in our study.

\section{Conclusion}

Scrotal pain is common condition. It can be due to nonspecific cause or due to serious organ threatening condition so timely evaluation and treatment is very important. 


\section{References}

1. Gordhan CG, SN Hossein. Scrotal pain: Evaluation and management. Korean J Urol 2015;56:3-11.

2. Sherieka W, Hoffmann B. Emergency ultrasound of acute scrotal pain. European Journal of Emergency Medicine 2015; 22:2-9.

3. Davis BE, Noble MJ, Weigel JW, Foret JD, Mebust WK. Analysis and management of chronic testicular pain. J Urol 1990;143:936-9.

4. Yusuf GT, Sidhu PS A review of ultrasound imaging in scrotal emergencies. J Ultrasound 2013; 16:171-178.

5. Ringdahl E, Teague L. Testicular torsion. Am Fam Physician 2006;74:1739-43.

6. Boettcher $\mathrm{M}$, Bergholz $\mathrm{R}$, Krebs $\mathrm{TF}$ et al. Differentiation of epididymitis and appendix testis torsion by clinical and ultrasound signs in children. Urology 2013;82:899-904.
7. Joo JM, Yang SH, Kang TW, Jung JH, Kim SJ, Kim KJ. Acute epididymitis in children: the role of the urine test. Korean J Urol 2013;54:135-8.

8. Levine L. Chronic orchalgia: evaluation and discussion of treatment options. Ther Adv Urol 2010;2:209-14.

9. Blaivas M, Sierzenski P, Lambert M. Emergency evaluation of patients presenting with acute scrotum using bedside ultrasonography. Acad Emerg Med 2001; 8:90-93.

10. Blaivas M, Brannam L. Testicular ultrasound. Emerg Med Clin North Am 2004; 22:723-748.

11. Galejs LE. Diagnosis and treatment of the acute scrotum. Am Fam Physician 1999; 59:817-824.

12. Yagil Y, Naroditsky I, Milhem J et al. Roleof Doppler ultrasonography in the triage of acute scrotum in the emergencydepartment. J Ultrasound Med 2010; 29:11-21. 